Check for updates

Cite this: RSC Adv., 2018, 8, 29071

Received 6th July 2018

Accepted 8th August 2018

DOI: $10.1039 / c 8 r a 05755 a$

rsc.li/rsc-advances

\section{Effect of the itinerant electron density on the magnetization and Curie temperature of $\mathrm{Sr}_{2} \mathrm{FeMoO}_{6}$ ceramics}

\author{
Jin-Feng Wang, (DD * Teng-Fei Shi, Zhao-Tong Zhuang, Qian-Qian Gao \\ and Yan-Ming Zhang
}

The itinerant electron density $(n)$ near the Fermi level has a close correlation with the physical properties of $\mathrm{Sr}_{2} \mathrm{FeMoO}_{6}$. Two series of single-phase $\mathrm{Sr}_{(2-y)} \mathrm{Na}_{y} \mathrm{FeMoO}_{6}(y=0.1,0.2,0.3)$ and $\mathrm{Sr}_{(2-y)} \mathrm{Na}_{y} \mathrm{Fe}_{(1-x)} \mathrm{Mo}_{(1+x)} \mathrm{O}_{6}(y$ $=2 x ; y=0.1,0.2,0.3)$ ceramics were specially designed and the itinerant electron density $(n)$ of them can be artificially controlled to be: $n=1-y$ and $n=1-y+3 x=1+0.5 y$, respectively. The corresponding crystal structure, magnetization and the ferromagnetic Curie temperature $\left(T_{C}\right)$ of two subjects were investigated systematically. The $\mathrm{X}$-ray diffraction analysis indicates that $\operatorname{Sr}_{(2-y)} \mathrm{Na}_{y} \mathrm{FeMoO}_{6}(y=0.1,0.2,0.3)$ have comparable Fe/Mo anti-site defect (ASD) content in spite of decreased $n$. However, a drastically improved Fe/Mo ASD can be observed in $\mathrm{Sr}_{(2-y)} \mathrm{Na}_{y} \mathrm{Fe}_{(1-x)} \mathrm{Mo}_{(1+x)} \mathrm{O}_{6}(y=2 x ; y=0.1,0.2,0.3)$ caused by the intrinsic wrong occupation of normal Fe sites with excess Mo. Magnetization-magnetic field $(M-H)$ behavior confirms that it is the Fe/Mo ASD not $n$ that dominantly determines the magnetization properties. Interestingly, approximately when $n \leq 0.9, T_{C}$ of $\operatorname{Sr}_{(2-y)} \mathrm{Na}_{y} \mathrm{FeMoO}_{6}(y=0.1,0.2,0.3)$ exhibits an overall increase with decreasing $n$, which is contrary to the $T_{C}$ response in electron-doped SFMO. Such abnormal $T_{C}$ is supposed to relate with the ratio variation of $n(\mathrm{Mo}) / n(\mathrm{Fe})$. Moreover, when $n \geq 1, T_{\mathrm{C}}$ of $\mathrm{Sr}_{(2-y)} \mathrm{Na}_{y} \mathrm{Fe}_{(1-x)} \mathrm{Mo}_{(1+x)} \mathrm{O}_{6}(y=2 x ; y=0.3)$ exhibits a considerable rise of about $75 \mathrm{~K}$ over that of $\mathrm{Sr}_{(2-y)} \mathrm{Na}_{y} \mathrm{Fe}_{(1-x)} \mathrm{Mo}_{(1+x)} \mathrm{O}_{6}(y=2 x ; y=0.1)$, resulting from improved $n$ caused by introducing excess Mo into $\mathrm{Sr}_{(2-y)} \mathrm{Na}_{y} \mathrm{FeMoO}_{6}$. Maybe, our work can provide an effective strategy to artificially control $n$ and ferromagnetic $T_{C}$ accordingly, and provoke further investigation on the FeMo-baseddouble perovskites.

\section{Introduction}

A strongly correlated electron system $\mathrm{Sr}_{2} \mathrm{FeMoO}_{6}$ (SFMO) has been extensively investigated due to its rich physical properties. Ferromagnetic SFMO material has a half-metallic nature with a $100 \%$ spin polarization at the ground state, an attractive lowfield magnetoresistance response and a high $T_{\mathrm{C}}$ of approximately $415 \mathrm{~K}^{\mathbf{1 - 5}}$ Materials combing such functional properties are very rare. Therefore, SFMO has become one of the most promising materials for its scientific research values and potential technological applications. ${ }^{\mathbf{2}, \mathbf{6 - 8}}$

The electron configuration in SFMO near the Fermi level is basically described by the localized spin-up electrons of the $3 \mathrm{~d}^{5}$ $\left(\mathrm{Fe}^{3+}\right)$ and the itinerant spin-down electron of the $4 \mathrm{~d}^{1}\left(\mathrm{Mo}^{5+}\right)$ shared by the $\mathrm{Fe}^{3+}\left(\mathrm{t}_{2 \mathrm{~g}} \downarrow\right)-\mathrm{O}(2 \mathrm{p})-\mathrm{Mo}^{5+}\left(\mathrm{t}_{2 \mathrm{~g}} \downarrow\right)$ subband., ${ }^{2,3,9,10}$ The $\mathrm{Fe}^{3+}\left(3 \mathrm{~d}^{5}: \mathrm{S}=5 / 2\right)$ antiferromagnetically interacts with $\mathrm{Mo}^{5+}\left(4 \mathrm{~d}^{1}: \mathrm{S}=1 / 2\right)$, leading to an ideal saturated magnetization $\left(M_{\mathrm{S}}\right)$ of $4 \mu_{\mathrm{B}} /$ f.u. However, a much smaller $M_{\mathrm{s}}$ than $4 \mu_{\mathrm{B}} /$ f.u. is commonly observed in experiments because of the appearance

College of Physics and Materials Science, National Demonstration Center for Experimental Physics Education, Henan Normal University, Henan Key Laboratory of Photovoltaic Materials, Xinxiang 453007, China.E-mail: jfwang@htu.edu.cn of the Fe/Mo anti-site defect (ASD, i.e., the Fe occupies the Mo site and vice versa). ${ }^{11-13}$ It has been widely accepted that ASD mainly control the magnetization. ${ }^{\mathbf{1 1 , 1 3 , 1 4}}$ The itinerant electron contributed by the $\mathrm{Mo}^{5+}$ cation mediates the ferromagnetic coupling between neighbour Fe cations via a double-exchangelike model.,15-17 The strengthen of the ferromagnetic interaction has a direct correlation with the density of the electron at the conduction band, which can be modified effectively by electron-doping or hole-doping. For example, substituting the divalent $\mathrm{A}^{2+}$ ions in $\mathrm{A}_{2} \mathrm{FeMoO}_{6}\left(\mathrm{~A}: \mathrm{Ca}^{2+} / \mathrm{Sr}^{2+} / \mathrm{Ba}^{2+}\right.$ ) with the trivalent ions (such as $\mathrm{La}^{3+}, \mathrm{Nd}^{3+}, \mathrm{Sm}^{3+}$ etc.), a significantly improved $T_{\mathrm{C}}$ can be observed due to the increased electron density caused by electron injection into the conduction band.,16,18-24 Contrary to the $T_{\mathrm{C}}$ response, the Fe/Mo ordering degree, saturated magnetization and magnetoresistance are reduced., 9,181,25,26 However, reports of the effect of hole-doping on the carrier density and the physical properties are relatively rare. Moreover, there are still controversies on the influence of the hole doping on the carrier density, ASD, magnetization and $T_{\mathrm{C}}$. Mainly two aspects can be addressed. One is that, $T_{\mathrm{C}}$ should lessen caused by a decreased carrier density near the Fermi level, which is contributed by the holedoping effect. ${ }^{27,28}$ However, an improved $T_{\mathrm{C}}$ is reported in 
some literatures. ${ }^{29,30}$ Second, it is failure to reaches a consensus over the effect of hole-doping on the ASD and magnetization. Ksubstituted $\mathrm{A}_{2} \mathrm{FeMoO}_{6}$ systems $\left(\mathrm{A}: \mathrm{Sr}^{2+} / \mathrm{Ba}^{2+}\right.$ ) show an improved ASD concentration, thus a decreased magnetization. ${ }^{27}$ On the contrary, Zhang et al. hold that Na-doping reduces the ASD content of SFMO and optimizes magnetization at a certain substitution level. ${ }^{29}$ However, Yoshida et al. claimed that, ASD is insensitive to the Na-content in $\mathrm{Ba}_{2-x} \mathrm{Na}_{x} \mathrm{FeMoO}_{6}$, the decreased magnetization has no relation with the ASD content nor carrier density, but caused by the increased $\mathrm{O}_{2 p}$ ligand hole. ${ }^{31}$ Therefore, it is necessary to investigate itinerant electron density, magnetization and Curie temperature by an appropriate hole-doping in SFMO.

Actually, the itinerant electron at the conduction band in SFMO is vital to maintain the ferromagnetic characteristic. For example, partially replacing $\mathrm{Mo}^{5+}$ cation with $\mathrm{W}^{6+}$ in $\mathrm{Sr}_{2}-$ $\mathrm{FeMo}_{1-x} \mathrm{~W}_{x} \mathrm{O}_{6}$ can greatly weaken the ferromagnetic interaction, and even transit a ferromagnetic into an antiferromagnetic coupling, ${ }^{32,33}$ thus a suppressed $T_{\mathrm{C}}$ is observed. It is noted that the itinerant electron is contributed by $\mathrm{Mo}^{5+}$ cation. Hence, it may be an effective method to increase the itinerant electron density by introducing excess Mo into SFMO. As mentioned above, hole-doped $\mathrm{A}_{2} \mathrm{FeMoO}_{6}(\mathrm{~A}: \mathrm{Sr} / \mathrm{Ba}$ ) systems demonstrate a decreased itinerant electron density near the Fermi level due to electron extraction effect from the conduction band. ${ }^{27-29}$ Therefore, introducing an appropriate excess Mo into holedoped SFMO possibly not only offset the decreased carrier density but also has a surplus of carrier density. Then the magnetization and $T_{\mathrm{C}}$ will be accordingly manipulated. The corresponding research works are relatively rare. ${ }^{30}$ Hence, it is interesting to conduct further research on these subjects.

In this work, single-phased Na-doped $\mathrm{Sr}_{(2-y)} \mathrm{Na}_{y} \mathrm{FeMoO}_{6}(y=$ $0.1,0.2,0.3)$ ceramics were prepared, here the nominal itinerant electron density $(n)$ can be expressed: $n=1-y$, where $n$ decrease with increasing Na-content $(y)$. For comparison, pure $\mathrm{Sr}_{(2-y)} \mathrm{Na}_{y} \mathrm{Fe}_{(1-x)} \mathrm{Mo}_{(1+x)} \mathrm{O}_{6}$ with an excess Mo content for the same $y$ values $(y=2 x ; y=0.1,0.2,0.3)$ were also synthesized, here the nominal $n$ is calculated to be $n=1-y+3 x=1+0.5 y$, where $n$ in $\operatorname{Sr}_{(2-y)} \mathrm{Na}_{y} \mathrm{Fe}_{(1-x)} \mathrm{Mo}_{(1+x)} \mathrm{O}_{6}$ system increase with increasing Na-content $(y)$. The itinerant electron density, and corresponding crystal structure, chemical states, magnetization and $T_{\mathrm{C}}$ of two objective systems are investigated systematically and comparatively.

\section{Experimental}

For simplicity, singled phased $\mathrm{Sr}_{(2-y)} \mathrm{Na}_{y} \mathrm{FeMoO}_{6}(y=0.1,0.2$, 0.3) ceramics were labeled as C1, C3 and C5. Pure $\operatorname{Sr}_{(2-y)} \mathrm{Na}_{y^{-}}$ $\mathrm{Fe}_{(1-x)} \mathrm{Mo}_{(1+x)} \mathrm{O}_{6}(y=2 x ; y=0.1,0.2,0.3)$ samples were identified as C2, C4 and C6, respectively. Each group of (C1, C2), (C3, C4), (C5, C6) has the same $y$ value. The above-mentioned compositions were synthesized by a solid-state reaction method described as elsewhere. ${ }^{34,35}$ The typical experimental process can be expressed as below. First, all the raw materials of $\mathrm{SrCO}_{3}$ (99.9\%), $\mathrm{Na}_{2} \mathrm{CO}_{3}$ (99.9\%), $\mathrm{Fe}_{2} \mathrm{O}_{3}$ (99.9\%), and $\mathrm{MoO}_{3}$ $(99.5 \%)$ were calculated, dried and weighed according to the nominal chemical formulas, mixed homogeneously by ball milling in ethanol for 24 hours, and dried in air at $90{ }^{\circ} \mathrm{C}$ for 10 hours. The dried mixtures were calcined at $800{ }^{\circ} \mathrm{C}$ for 10 hours in air. Then, the calcined powders were ball milled in ethanol again for another 24 hours, and the slurry was dried and pressed into small thin pellets $(10 \times 1 \mathrm{~mm})$. Finally, the pellets were sintered at $1280{ }^{\circ} \mathrm{C}$ for 12 hours in a reducing atmosphere of a mixed gas of $5 \% \mathrm{H}_{2} / 95 \%$ Ar. Actually, in order to prepared high-quality ceramics, a sintering process was conducted with different experimental parameters. Here, we fix at 5\% $\mathrm{H}_{2} / 95 \%$ Ar mixed gas, and then we carried out a systemic investigation by varying the sintering temperature $\left(1050-1623{ }^{\circ} \mathrm{C}\right)$, the flowing rate of the gases $\left(40-90 \mathrm{ml} \mathrm{min}^{-1}\right)$ and the sintering time (30-3 h) for sintering. Briefly, in our experiments, it is found that too high or too low sintering temperature $\left(>1623{ }^{\circ} \mathrm{C},<1050\right.$ ${ }^{\circ} \mathrm{C}$ ) generally lead to second phases. On the other hand, with moderate sintering temperature $\left(1280^{\circ} \mathrm{C}\right)$, single phase $\mathrm{C} 1-\mathrm{C} 6$ ceramics can be obtained readily. In fact, the flowing rate of the reducing gas is also a decisive factor at a given experimental condition for the fixed $\mathrm{H}_{2} / \mathrm{Ar}$. For instance, phase-pure samples of SFMO can only be synthesized using 40-90 $\mathrm{ml} \mathrm{min}^{-1}$ gas flow rates (5\% $\left.\mathrm{H}_{2} / 95 \% \mathrm{Ar}\right)$. Exceeding the upper limit $\left(90 \mathrm{ml} \mathrm{min}^{-1}\right)$ not only diminishes the ordering degree but also introduces over reduced impurity phases such as $\mathrm{SrMoO}_{3}$, metal Fe and so on, whereas using less than the lower limit $\left(40 \mathrm{ml} \mathrm{min}^{-1}\right)$ leads to $\mathrm{SrMoO}_{4}$ as the second phase. Based on these results, with a fixed sintering temperature of $1280{ }^{\circ} \mathrm{C}$, reducing gas of $5 \% \mathrm{H}_{2}$ /

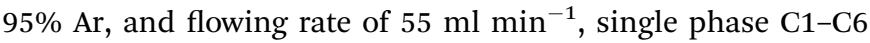
ceramics are obtained by only varying the sintering time (3-30 h).

The structure of the compositions was characterized by X-ray diffraction patterns (XRD, Bruker D8 Discover). The chemical states of the $\mathrm{Fe}$ and Mo cations were investigated by X-ray photoelectron spectroscopy (XPS, Thermo Scientific K-Alpha). The magnetic data were obtained using a superconducting vibrating sample magnetometer (VSM) and a physical property measurements system (PPMS Quantum Design, 2001NUGC).

\section{Results and discussions}

Fig. 1(a) shows all the XRD patterns of C1-C6 ceramics. One can see clearly that all the diffracted peaks of C1-C6 are well consistent with the double perovskite structure with a tetragonal phase and a space group of $I 4 / m^{3,36,37}$ and no second phases are identified. The superstructure diffraction peak (101) located at approximately $19.8^{\circ} \mathrm{C}$ has a close correlation with the Fe/Mo ordering degree (Fig. 1(b)). As for the normalized XRD, the peak intensity of (101) has a positive correlation with Fe/Mo ordering degree, whereas a negative correlation with Fe/Mo ASD content. The (101) peak intensity of C1, C3 and C5 is comparable, indicating Fe/Mo ASD content of them are almost equivalent This result implies that Fe/Mo ASD content in Nadoped $\mathrm{Sr}_{(2-y)} \mathrm{Na}_{y} \mathrm{FeMoO}_{6}(y=0.1,0.2,0.3)$ systems are insensitive to both Na-doped concentration $(y)$ and decreased $n$. The observations are in accordance with the published data in $\mathrm{Na}$ doped $\mathrm{Ba}_{2-x} \mathrm{Na}_{x} \mathrm{FeMoO}_{6},{ }^{31}$ but quite different from other similar hole-doped systems. ${ }^{27,29}$ Taking $\mathrm{Sr}_{2-x} \mathrm{Na}_{x} \mathrm{FeMoO}_{6}$ for example, the Fe/Mo ASD content decreases gradually with 


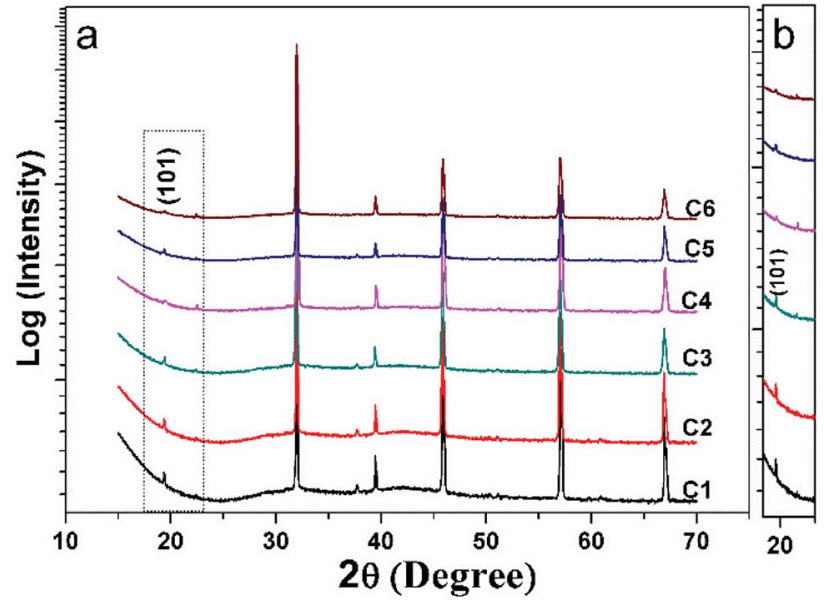

Fig. 1 (a) X-ray diffraction patterns of C1-C6; (b) the locally enlarged XRD images of (a).

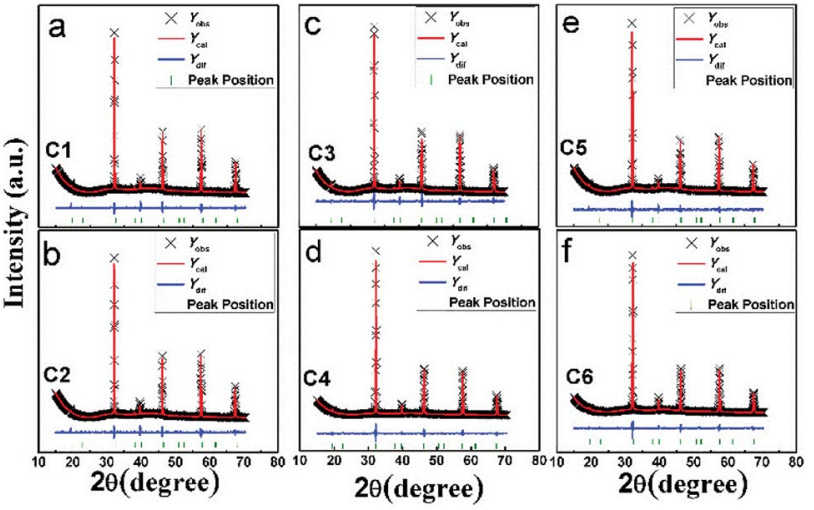

Fig. 2 Observed (crosses), calculated (red solid line), and difference (blue solid line) XRD patterns of $\mathrm{C} 1-\mathrm{C} 6$. The bottom green bars indicate the peak positions.

increasing Na-doped concentration. It is suggested that, $\mathrm{Fe} / \mathrm{Mo}$ ASD content negatively relate to the charge difference between Fe and Mo cations, which may decrease because of a larger charge difference caused by the injection of the hole into the conduction band (Fig. 2).

A comparable peak intensity of (101) of C1 and C2 $\left(\operatorname{Sr}_{(2-y)^{-}}\right.$ $\mathrm{Na}_{\mathrm{y}} \mathrm{Fe}_{(1-x)} \mathrm{Mo}_{(1+x)} \mathrm{O}_{6} ; y=2 x ; y=0.1$ ) implies that small amount
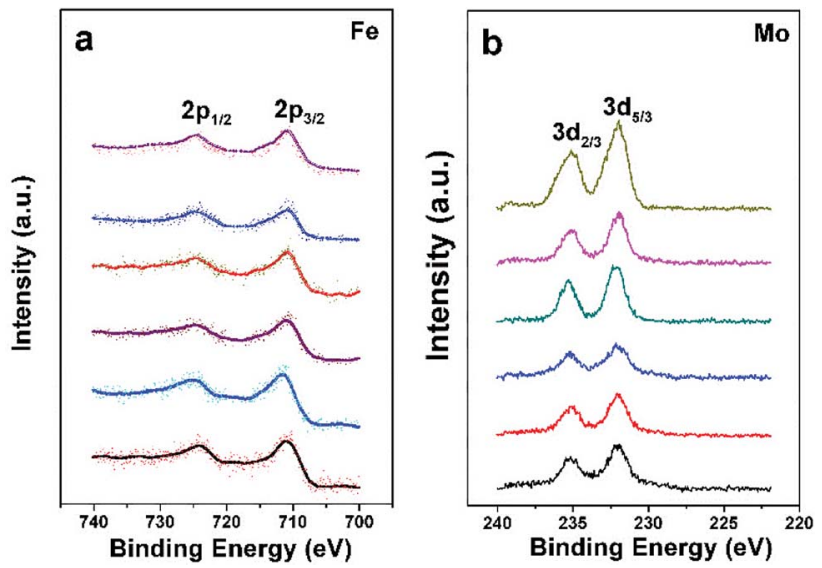

Fig. 3 X-ray photoelectron spectra of the Fe (a) and Mo (b) cations of C1-C6.

of excess Mo has a negligent function on the Fe/Mo ordering degree. However, the suppression of the excess Mo content $(x)$ on the Fe/Mo ordering degree become obvious when $y$ increases to 0.1 (C4) and 0.15 (C6), as indicated in Fig. 1(b). The possible reason may lies in that replacing normal Fe site with excess Mo in $\mathrm{Sr}_{(2-y)} \mathrm{Na}_{\mathrm{y}} \mathrm{Fe}_{(1-x)} \mathrm{Mo}_{(1+x)} \mathrm{O}_{6}$ will bring about intrinsic $\mathrm{Fe} / \mathrm{Mo}$ ASD, thus an increased Fe/Mo ASD in C4 and C6 are observed. In order to give a quantitative estimation of Fe/Mo ASD concentration of the samples, all the X-ray profiles were investigated further using the refinements work, based on the tetragonal $I 4 / \mathrm{m}$ space group. It is noted that $\mathrm{Sr} / \mathrm{Na}$ atoms were located at $4 \mathrm{~d}(0,0.5,0.25)$ sites, Fe at $2 \mathrm{a}(0,0,0)$, Mo at $2 \mathrm{~b}(0,0$, $0.5)$ positions, and oxygen atoms at $4 \mathrm{e} \mathrm{O} 1(0,0, z)$ and $\mathrm{O} 2$ at $8 \mathrm{~h}$ $(x, y, 0)$. The refined results are shown in the Table 1 . With increasing Na-content $(y)$, C1-C5 show a comparable ASD content of approximately $14 \%$, but a gradual rising ASD can be observed in C2-C6. This is consistent with the indications by the Fig. 1. The cell parameters of both C1-C5 and C2-C6 decrease slightly due to smaller ionic sizes of $\mathrm{Na} / \mathrm{Mo}$ than $\mathrm{Sr} / \mathrm{Fe}$ cations, manifesting the actual occurrence of the designed elements substitutions.

The chemical states of Fe and Mo cations in SFMO are closely related to magnetic behaviors and other physical properties. Thus the chemical states of Fe and Mo cations are confirmed by the XPS as shown in Fig. 3(a) and (b), respectively. From Fig. 3(a), one can see clearly that $\mathrm{Fe} 2 \mathrm{p}_{3 / 2}$ and $\mathrm{Fe} 2 \mathrm{p}_{1 / 2}$ of C1-C6

Table 1 The refinement results of the $\mathrm{C} 1-\mathrm{C} 6$ ceramics

\begin{tabular}{|c|c|c|c|}
\hline Sample I & $\mathrm{C} 1(y=0.1)$ & $\mathrm{C} 3(y=0.2)$ & $\mathrm{C} 5(y=0.3)$ \\
\hline$a=b / c(\AA)$ & $5.5652(0) / 7.8952(7)$ & $5.5538(6) / 7.8973(3)$ & $5.5536(1) / 7.8801(8)$ \\
\hline Cell volume $\left(\AA^{3}\right)$ & 244.528 & 243.596 & 243.042 \\
\hline $\mathrm{ASD} \%$ & 14.(8) & 13.(9) & 14.(4) \\
\hline$R_{\mathrm{W}} / R_{\mathrm{p}}$ & $10.46 / 6.35$ & $10.51 / 6.57$ & $10.28 / 6.30$ \\
\hline Sample II & $\mathrm{C} 2(y=0.1=2 x)$ & $\mathrm{C} 4(y=0.2=2 x)$ & $\mathrm{C} 6(y=0.3=2 x)$ \\
\hline$a=b / c(\AA)$ & $5.5651(4) / 7.8949(9)$ & $5.5516(3) / 7.8978(5)$ & $5.5508(3) / 7.8709(7)$ \\
\hline Cell volume $\left(\AA^{3}\right)$ & 244.514 & 243.412 & 242.513 \\
\hline $\mathrm{ASD} \%$ & 15.(3) & 18.(3) & 25.(3) \\
\hline$R_{\mathrm{W}} / R_{\mathrm{p}}$ & $10.46 / 6.72$ & $10.32 / 6.62$ & $10.31 / 6.38$ \\
\hline
\end{tabular}



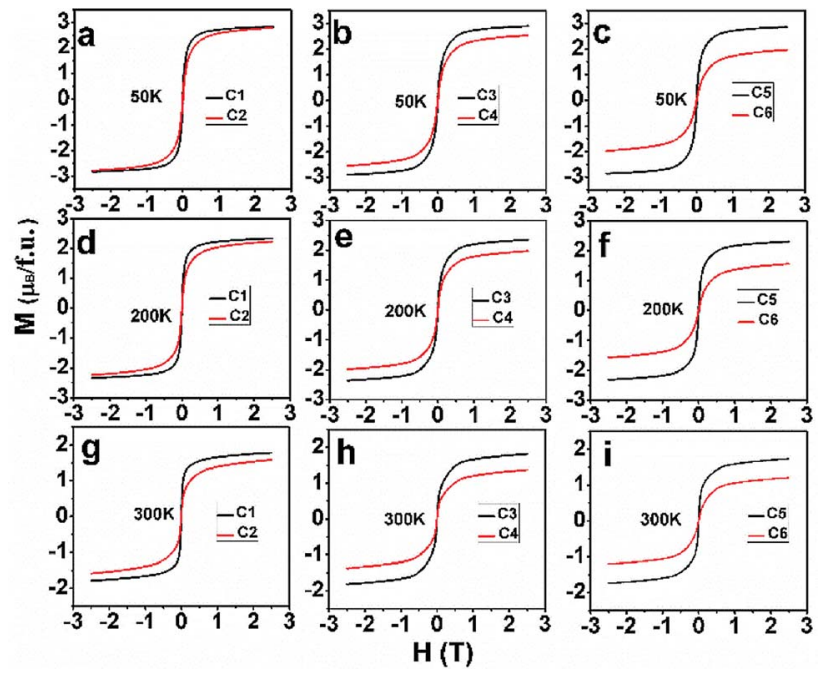

Fig. $4 M-H$ curves of $\mathrm{C} 1-\mathrm{C} 6$ at $50 \mathrm{~K}(\mathrm{a})-(\mathrm{c})$; at $200 \mathrm{~K}(\mathrm{~d})-(\mathrm{f})$ and at $300 \mathrm{~K}(\mathrm{~g})-(\mathrm{h})$.

compositions are located at $c a .710 .5 \mathrm{eV}$ and $724.5 \mathrm{eV}$, with a spin-energy separation of $14 \mathrm{eV}$, which indicates the Fe cations in C1-C6 mainly are $3+{ }^{38,39}$ The double peaks contributed by the $\operatorname{Mo3d}_{5 / 3}$ and Mo3d $\mathrm{d}_{2 / 3}$ caused by spin-orbit coupling can be observed in Fig. 3(b). Except for that, there are no other shoulder peaks, which is different from the XPS data in SFMO systems in the literatures. ${ }^{38,40}$ The location at binding energy of $\operatorname{Mo}_{5 / 3}$ and Mo3d $\mathrm{d}_{2 / 3}$ peaks shows a weak shift, within the range of $232.2-231.5 \mathrm{eV}$ and 234.8-235.4 eV. Actually, all those Mo-3d XPS experimental data are in accordance with the reports, it demonstrates that the chemical state of the Mo cations of C1C6 mainly is $5+$ valences. ${ }^{17,40,41}$ The XPS results demonstrate that both Na-doped content $(y)$ and excess-Mo $(x)$ content play a negligible role of the chemical states of Fe and Mo cations.

After confirm the chemical states of Fe and Mo cations, it is more reasonable to discuss magnetic behavior of C1-C6. $M-H$ curves of C1-C6 measured at $50 \mathrm{~K}, 200 \mathrm{~K}$ and $300 \mathrm{~K}$ are plotted in Fig. 4(a)-(c), (d)-(f) and (g)-(i), respectively. All the $M-H$ curves present well-saturated magnetic hysteresis loops with a very small magnetic coercivity, indicating a soft ferromagnetic feature. ${ }^{3,42,43}$ The macroscopic ferromagnetic natures of six compositions have a negligent relation with the Na-doped content $(y)$ and excess Mo content $(x=0.5 y)$. In order to deeply investigate the influence of Na-doped content $(y)$ on the magnetization, $M_{\mathrm{s}}$ versus Na-doped content $(y)$ at $50 \mathrm{~K}, 200 \mathrm{~K}$ and $300 \mathrm{~K}$ are shown in Fig. 5(a)-(c). From Fig. 5(a), mainly two aspects of $M_{\mathrm{s}}$ with $y$ can be addressed. First, $\mathrm{C} 1, \mathrm{C} 3$ and $\mathrm{C} 5$ have comparable $M_{\mathrm{S}}$ values of 2.83, 2.9 and $2.86 \mu_{\mathrm{B}} /$ f.u., respectively. This observation indicates that Na-doped content $(y)$ have not an obvious function on the $M_{\mathrm{s}}$ values. Second, as for a certain $y$ value, the $M_{\mathrm{s}}$ values of $\mathrm{C} 2, \mathrm{C} 4$ and $\mathrm{C} 6$ ceramics are less than that of the C1, C3 and C5, and $M_{\mathrm{S}}$ values of C2-C6 gradually decrease further with increasing $y$. This experimental result shows that introducing excess Mo into Na-doped SFMO has a negative contribution to the $M_{\mathrm{s}}$. However, the fundamentally causes for the observations are unclear, and it is necessary to originally discuss the possible reasons.

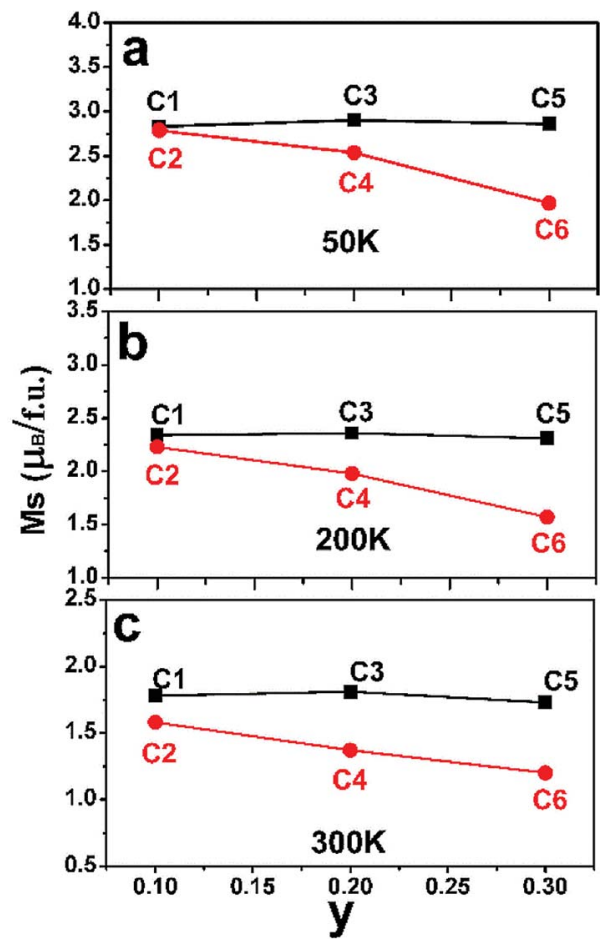

Fig. 5 The Na-content $(y)$ dependent saturated magnetization $\left(M_{\mathrm{s}}\right)$ values of $\mathrm{C} 1-\mathrm{C} 6$ at $50 \mathrm{~K}$ (a); $200 \mathrm{~K}$ (b) and $300 \mathrm{~K}$ (c).

Based on previous studies, mainly two alternative explanations are proposed for the magnetization in FeMo-based double perovskites. One is $\mathrm{Fe} / \mathrm{Mo}$ ASD effect originated from the wrong occupation of Fe sites with the Mo cation or vice versa. Once Fe/ Mo ASD occurs, giving rise to antiferromagnetic - $\mathrm{Fe}-\mathrm{O}-\mathrm{Fe}-\mathrm{O}-$ patches and paramagnetic - Mo-O-Mo-O- patches between ordering ferromagnetic domains. Actually, a great deal of experimental results confirmed that both patches can greatly weaken the magnetization in FeMo-based double perovskites, ${ }^{10-13}$ in the other explanation, itinerant electron density $n$ at the conduction band is presumed to has a negative correlation with the magnetization. ${ }^{29}$ Because localized electrons of $\mathrm{Fe}^{3+}\left(3 \mathrm{~d}^{5}, \mathrm{~S}=5 / 2\right)$ antiferromagnetically couple with the itinerant electron of $\mathrm{Mo}^{5+}\left(4 \mathrm{~d}^{1}, \mathrm{~S}=1 / 2\right)$, resulting in an ideal $M_{\mathrm{S}}$ of $4 \mu_{\mathrm{B}} / \mathrm{f}$.u. If itinerant electron density $n$ is less than 1 , the $M_{\mathrm{S}}$ will be more than $4 \mu_{\mathrm{B}} /$ f.u., otherwise less than $4 \mu_{\mathrm{B}} /$ f.u. According to the second explanation, the itinerant electron density $n$ of C1C6 are estimated and shown in Table 2. Herein, supposing $100 \% \mathrm{Fe} / \mathrm{Mo}$ ordering degree in $\mathrm{C} 1-\mathrm{C} 6$, the perfect saturated magnetization $\left(M_{\mathrm{S}}(\mathrm{I})\right)$ values are calculated for C1-C6 through a magnetic double-exchange-like mechanism.

The variance of Fe/Mo ASD (Fig. 1, Table 1) and $n$ (Table 2) have been investigated. Then, the functions of them on magnetization are estimated by combing experimental $M_{\mathrm{s}}$ data as shown in Fig. 5 . Supposing the $M_{\mathrm{s}}$ were mainly controlled by the itinerant electron density $n$, then a gradual increased $M_{\mathrm{s}}$ will be observed from C1 to C5 since a comparable ASD content of C1C5. However, the measured $M_{\mathrm{s}}$ in Fig. 5 of C1-C5 show equivalent $M_{\mathrm{S}}$ values. It indicates that the Fe/Mo ASD content, not $n$ control 
Table 2 The itinerant electron density $n$ and the ideal saturated $M_{\mathrm{s}}(\mathrm{I})$ of $\mathrm{C} 1-\mathrm{C} 6$

\begin{tabular}{|c|c|c|c|}
\hline $\begin{array}{l}\text { Sample I } \\
n=1-y \\
M_{\mathrm{s}}(\mathrm{I})\left(\mu_{\mathrm{B}} / \text { f.u. }\right)\end{array}$ & $\begin{array}{l}\text { C1 }(y=0.1) \\
0.9 \\
4.1\end{array}$ & $\begin{array}{l}\text { C3 }(y=0.2) \\
0.8 \\
4.2\end{array}$ & $\begin{array}{l}\text { C5 }(y=0.3) \\
0.7 \\
4.3\end{array}$ \\
\hline Sample II & $\begin{array}{l}\mathrm{C} 2 \\
(y=0.1=2 x)\end{array}$ & $\begin{array}{l}\text { C4 } \\
(y=0.2=2 x)\end{array}$ & $\begin{array}{l}\text { C6 } \\
(y=0.3=2 x)\end{array}$ \\
\hline$n=1+0.5 y$ & 1.05 & 1.1 & 1.15 \\
\hline$M_{\mathrm{s}}(\mathrm{I})\left(\mu_{\mathrm{B}} /\right.$ f.u. $)$ & 3.95 & 3.9 & 3.85 \\
\hline
\end{tabular}

the $M_{\mathrm{s}}$ behavior dominantly. Introducing excess Mo into Nadoped SFMO leads to an improved $n$, thus a decreased $M_{\mathrm{s}}$ (I) (Table 2). Although varied trend of the $M_{\mathrm{S}}$ of C2-C6 is consistent with both $M_{\mathrm{S}}$ (I) (Table 2) and Fe/Mo ordering degree (Fig. 1, Table 1), the amplitude of variation of $M_{\mathrm{S}}$ (I) (Table 2) is much less than the experimental $M_{\mathrm{S}}$ in Fig. 5(a) and the $M_{\mathrm{S}}$ (I) values are greater than the experimental $M_{\mathrm{s}}$. For example, $M_{\mathrm{S}}$ (I) of C6 is $3.85 \mu_{\mathrm{B}} /$ f.u.(Table 2), which is almost two times that of the experimental $M_{\mathrm{S}}\left(1.97 \mu_{\mathrm{B}} /\right.$ f.u. $)$ in Fig. 5(a). This indicates that $n$ doesn't have a sufficient capability to affect the magnetization behavior in SFMO. On the other words, the significant discrepancy between $M_{\mathrm{s}}$ (I) and $M_{\mathrm{s}}$ is because of the negative effect of ASD on the magnetization. Actually, our experimental observations reveal mainly contribution of Fe/Mo ASD to the magnetization, which are in accordance with the experimental results reported in literatures. ${ }^{\mathbf{1 3 , 1 4 , 4 4}}$

In order to investigate the effect of $n$ on $T_{\mathrm{C}}$ of C1-C6, fieldcooling (200 Oe) magnetization as a dependence of temperature $(M-T)$ curves of C1-C6 are present in Fig. 6 . With increasing temperature, the $M-T$ curves show an abrupt decrease, implying a transition from ferromagnetic to paramagnetic state. ${ }^{\mathbf{3 , 1 4 , 1 8 , 2 1}}$ The corresponding ferromagnetic $T_{\mathrm{C}}$ can be established from the inflection point of $M-T$ curves.

The $T_{\mathrm{C}}$ values of C1-C6 are present in Fig. 7. As discussed in the introduction, a considerable high $T_{\mathrm{C}}$ of SFMO origins from the strong magnetic interactions via a double-exchange-like mechanism. Ferromagnetic coupling strengthen is proposed to be mediated by $n$. Actually, $T_{\mathrm{C}}$ indeed can be significantly
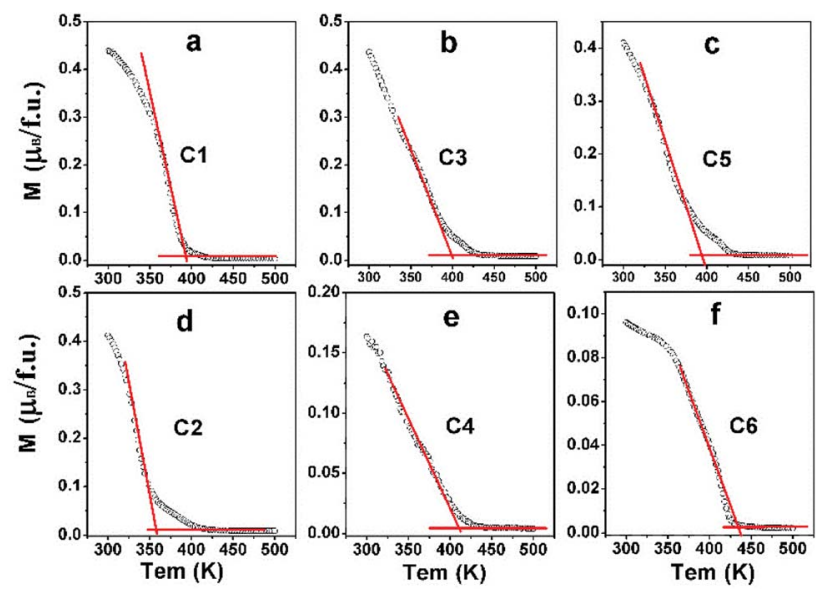

Fig. 6 Magnetization-temperature $(M-T)$ curves measured at $200 \mathrm{Oe}$ of C1 (a); C3 (b); C5 (c); C2 (d); C4 (e) and C6 (f).

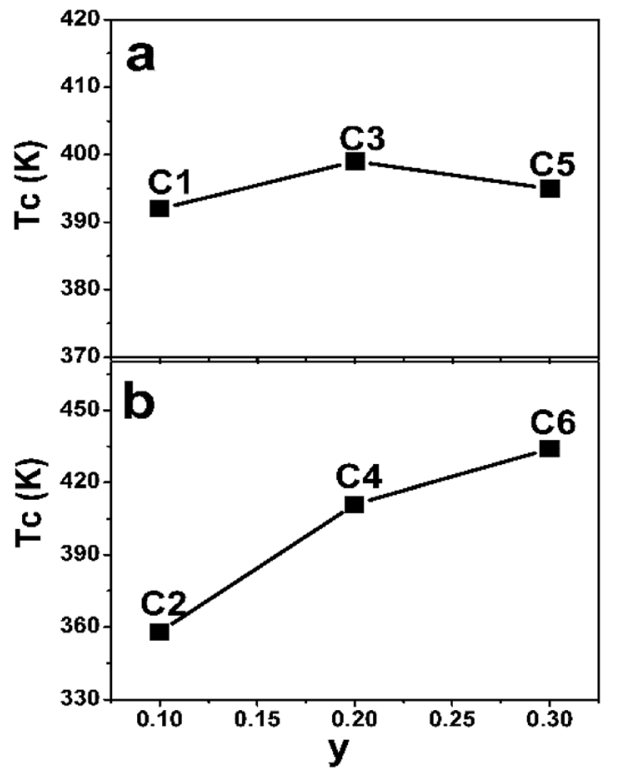

Fig. 7 The Curie temperature $\left(T_{C}\right)$ of $\mathrm{C} 1-\mathrm{C} 5(\mathrm{a})$ and $\mathrm{C} 2-\mathrm{C} 6(\mathrm{~b})$.

improved by electron doped FeMo-based double perovskites, ${ }^{16,18-22,45,46}$ and it is noted that the theoretical $n$ of such subjects investigated are greater than 1 . And $T_{\mathrm{C}}$ values have a monotonously positive correlation with $n$ due to the electron band filling effect. However, $T_{\mathrm{C}}$ of Na-doped C1-C5 shows a nonmonotonic variation, but an overall improvement with the Na-content $(y)$ (Fig. 7(a)). It indicates that $T_{\mathrm{C}}$ of Na-doped SFMO (C1-C5) don't decrease but improve with decreasing theoretical $n$, presenting a reverse tendency with that of electron-doped FeMo-based double perovskites. Similar observations are found in $\left(\mathrm{La}_{1-x} \mathrm{Ca}_{x}\right) \mathrm{MnO}_{3}$ and Na-doped SFMO literatures. ${ }^{29,30,47}$ Moreover, it seems that $T_{\mathrm{C}}$ of Na-doped SFMO likely to increase when approximately $n \leq 0.9 .^{30}$ The composition dependent $T_{\mathrm{C}}$ can be explained based on the ratio variation of $n(\mathrm{Mo}) / n(\mathrm{Fe})$, where, $n$ express the density of states. ${ }^{47} T_{\mathrm{C}}$ may has a positive relation with $n(\mathrm{Mo})$ but a negative relation with $n(\mathrm{Fe})$. Possibly, when $n \leq 0.9$, $n(\mathrm{Mo})$ decrease much more slowly than the $n(\mathrm{Fe})$, resulting in a larger $n(\mathrm{Mo})$ than $n(\mathrm{Fe})$. Hence, C3 and C5 show improved $T_{\mathrm{C}}$ though a decrease of the total $n$ near the Fermi level. Actually, further theoretical calculation works will be conducted to confirm the speculation. As for C2-C6 (Fig. 7(b)), it is interesting to find that $T_{\mathrm{C}}$ dramatically improves with increasing $\mathrm{Na}(\mathrm{Mo})$ content in spite of the increased Fe/Mo ASD concentration (Fig. 1, Table 1). $T_{\mathrm{C}}$ of $\mathrm{C} 6$ reaches to $434 \mathrm{~K}$, showing a substantial improvement of $76 \mathrm{~K}$ compared with $\mathrm{C} 2(358 \mathrm{~K})$. Although a small local region of ferromagnetic coupling strength will decrease caused by introducing a small amount of Mo, accompanying increased $n$ will enhance the ferromagnetic interaction strength of the most of the normal Fe/Mo crystal lattices. Therefore, a macroscopic ferromagnetic coupling strength will be improved, manifesting by a significantly increased $T_{\mathrm{C}}$ value as shown in Fig. 7(b). It also indicates that $T_{\mathrm{C}}$ invariably relates with the total $n$ positively when theoretical $n$ is more than 1 . The efficient optimized $T_{\mathrm{C}}$ strategies have been also proposed in the electron doped FeMo-based perovskites. ${ }^{\text {16,18,21,46 }}$ 


\section{Conclusions}

We have prepared single-phased $\operatorname{Sr}_{(2-y)} \mathrm{Na}_{y} \mathrm{FeMoO}_{6}(y=0.1,0.2$, $0.3)$ and $\operatorname{Sr}_{(2-y)} \mathrm{Na}_{y} \mathrm{Fe}_{(1-x)} \mathrm{Mo}_{(1+x)} \mathrm{O}_{6}(y=2 x ; y=0.1,0.2,0.3)$ ceramics to artificially control $n$ of them to be $n=1-y$ and $n=$ $1-y+3 x=1+0.5 y$, respectively. We have systematically investigated the corresponding crystal structure, magnetization and the ferromagnetic $T_{\mathrm{C}}$ of two objectives. The main results can be concluded as follows: The XRD analysis indicates that $n$ has a negligent effect on Fe/Mo ASD content of $\operatorname{Sr}_{(2-y)} \mathrm{Na}_{y^{-}}$ $\mathrm{FeMoO}_{6}(y=0.1,0.2,0.3)$. However, a drastic improved Fe/Mo ASD can be observed in $\operatorname{Sr}_{(2-y)} \mathrm{Na}_{y} \mathrm{Fe}_{(1-x)} \mathrm{Mo}_{(1+x)} \mathrm{O}_{6}(y=2 x ; y=$ $0.1,0.2,0.3)$ due to the intrinsic wrong occupation of normal Fe site with Mo cation. All the $M-H$ behavior confirms that it is the Fe/Mo ASD not $n$ dominantly determines the magnetization property. Interestingly, approximately when $n \leq 0.9, T_{\mathrm{C}}$ of $\mathrm{Sr}_{(2-y)} \mathrm{Na}_{y} \mathrm{FeMoO}_{6}(y=0.1,0.2,0.3)$ increase on the whole with decreasing $n$, which is contrary to the $T_{\mathrm{C}}$ response in electrondoped SFMO. Such abnormal $T_{\mathrm{C}}$ possibly relates with the ratio variation of $n(\mathrm{Mo}) / n(\mathrm{Fe})$. Moreover, when $n \geq 1, T_{\mathrm{C}}$ of $\mathrm{Sr}_{(2-y)} \mathrm{Na}_{y} \mathrm{Fe}_{(1-x)} \mathrm{Mo}_{(1+x)} \mathrm{O}_{6}(y=2 x ; y=0.3)$ exhibits a significant rise of about $75 \mathrm{~K}$ over that of $\mathrm{Sr}_{(2-y)} \mathrm{Na}_{y} \mathrm{Fe}_{(1-x)} \mathrm{Mo}_{(1+x)} \mathrm{O}_{6}(y=2 x$; $y=0.1$ ), resulting from improved $n$ caused by introducing excess Mo into $\mathrm{Sr}_{(2-y)} \mathrm{Na}_{y} \mathrm{FeMoO}_{6}$. Maybe, our work can provide an effective strategy to artificially control $n$ and ferromagnetic $T_{\mathrm{C}}$ accordingly, and provoke further investigation on the FeMobased double perovskites.

\section{Conflicts of interest}

There are no conflicts to declare.

\section{Acknowledgements}

This work was supported by funds from the Natural Science Foundation of China (U1504107) and the Doctoral Scientific Research Foundation (qd15214).

\section{References}

1 K. I. Kobayashi, T. Kimura, H. Sawada, K. Terakura and Y. Tokura, Nature, 1998, 395, 677.

2 W. E. Pickett and J. S. Moodera, Phys. Today, 2001, 54, 39.

3 D. Serrate, J. De Teresa and M. Ibarra, J. Phys.: Condens. Matter, 2007, 19, 023201.

4 J. F. Wang, J. Zhang, B. Hu, Z. B. Gu and S. T. Zhang, J. Phys. D: Appl. Phys., 2014, 47, 445003.

5 J. F. Wang, B. Hu, J. Zhang, Z. B. Gu and S. T. Zhang, J. Alloys Compd., 2015, 621, 131.

6 S. Vasala and M. Karppinen, Prog. Solid State Chem., 2015, 43, 1.

7 I. Hussain, M. S. Anwar, J. W. Kim, K. C. Chung and B. H. Koo, Ceram. Int., 2016, 42, 13098.

8 Z. Du, H. Zhao, S. Li, Y. Zhang, X. Chang, Q. Xia, N. Chen, L. Gu, K. Swierczek, Y. Li, T. Yang and K. An, Adv. Energy Mater., 2018, 8, 1800062.
9 J. Navarro, J. Fontcuberta, M. Izquierdo, J. Avila and M. C. Asensio, Phys. Rev. B, 2004, 70, 054423.

10 S. Ray, A. Kumar, D. D. Sarma, R. Cimino, S. Turchini, S. Zennaro and N. Zema, Phys. Rev. Lett., 2001, 87, 097204.

11 L. L. Balcells, J. Navarro, M. Bibes, A. Roig, B. Martínez and J. Fontcuberta, Appl. Phys. Lett., 2001, 78, 781.

12 Y. H. Huang, J. Linden, H. Yamauchi and M. Karppinen, Chem. Mater., 2004, 16, 4337.

13 J.-F. Wang, X.-J. Xu, W.-J. Ji, S.-T. Zhang, J. Zhou, Z.-B. Gu, Y. Chen, G.-L. Yuan, S.-H. Yao and Y.-F. Chen, CrystEngComm, 2013, 15, 4601.

14 J.-F. Wang, Z.-T. Zhuang, T.-F. Shi, Q.-Q. Gao and Z.-J. Jia, Ceram. Int., 2018, 44, 8492.

15 O. N. Meetei, O. Erten, A. Mukherjee, M. Randeria, N. Trivedi and P. Woodward, Phys. Rev. B: Condens. Matter Mater. Phys., 2013, 87, 165104.

16 E. K. Hemery, G. V. M. Williams and H. J. Trodahl, Phys. Rev. B: Condens. Matter Mater. Phys., 2006, 74, 054423.

17 P. Kumar, N. K. Singh, G. Gupta and P. Singh, RSC Adv., 2016, 6, 22094.

18 J. Navarro, C. Frontera, L. Balcells, B. Martínez and J. Fontcuberta, Phys. Rev. B: Condens. Matter Mater. Phys, 2001, 64, 092411.

19 C. Frontera, D. Rubi, J. Navarro, J. L. García-Muñoz, J. Fontcuberta and C. Ritter, Phys. Rev. B: Condens. Matter Mater. Phys., 2003, 68, 012412.

20 D. Rubi, J. Appl. Phys., 2004, 95, 7082.

21 D. Rubi, C. Frontera, J. Fontcuberta, M. Wojcik, E. Jedryka and C. Ritter, Phys. Rev. B: Condens. Matter Mater. Phys, 2004, 70, 094405.

22 A. K. Azad, S. G. Eriksson, A. Khan, A. Eriksson and M. Tseggai, J. Solid State Chem., 2006, 179, 1303.

23 T. Saitoh, M. Nakatake, H. Nakajima, O. Morimoto, A. Kakizaki, S. Xu, Y. Moritomo, N. Hamada and Y. Aiura, Phys. Rev. B: Condens. Matter Mater. Phys., 2005, 72, 045107.

24 Q. Zhang, G. Y. Liu, Z. F. Xu, X. M. Feng and G. H. Rao, Phys. B, 2010, 405, 1428.

25 D. Rubi and J. Fontcuberta, J. Phys.: Condens. Matter, 2006, 18, 7991.

26 J. Wang, X. Song, Y. Li and Z. Zhuang, Ceram. Int., 2017, 43, 5585.

27 J. Kim, J. G. Sung, H. M. Yang and B. W. Lee, J. Magn. Magn. Mater., 2005, 290, 1009.

28 G. Kim, S. S. Lee, S. C. Wi, J. S. Kang, S. W. Han, J. Y. Kim, B. W. Lee, J. Y. Kim, H. J. Shin and B. G. Parr, J. Appl. Phys., 2006, 99, 677.

29 Q. Zhang, G. H. Rao, Y. G. Xiao, G. Y. Liu, Y. Zhang and J. K. Liang, Appl. Phys. A, 2006, 84, 459.

30 J. F. Wang, J. F. Wang, Y. M. Zhang and Y. M. Zhang, RSC Adv., 2017, 7, 26185.

31 K. Yoshida, Y. Fujii and H. Shimizu, J. Appl. Phys., 2005, 98, 103901.

32 D. Sanchez, J. A. Alonso, M. Garcia-Hernandea, M. J. Martinez-Lope and M. T. Casais, J. Phys.: Condens. Matter, 2005, 3673.

33 M. Iranmanesh, M. Lingg, M. Stir and J. Hulliger, RSC Adv., 2016, 6, 42069. 
34 J. F. Wang and Y. M. Zhang, Mater. Res. Bull., 2017, 90, 145. 35 J. F. Wang, Y. M. Zhang, C. Jie and M. Xu, J. Phys. D: Appl. Phys., 2017, 50, 305001.

36 D. Yang, R. J. Harrison, J. A. Schiemer, G. I. Lampronti, X. Liu, F. Zhang, H. Ding, Y. g. Liu and M. A. Carpenter, Phys. Rev. B: Condens. Matter Mater. Phys., 2016, 93, 024101.

37 A. M. Reyes, Y. Arredondo and O. Navarro, J. Phys. Chem. C, 2016, 120, 4048.

38 W. Ji, J. F. Wang, J. Xu, L. Jiao, J. Zhou, Y. B. Chen, Z. B. Gu, S. H. Yao, S. T. Zhang and Y. F. Chen, J. Phys. D: Appl. Phys., 2013, 46, 015001.

39 M. Retuerto, F. Jiménezvillacorta, M. J. Martínezlope, Y. Huttel, E. Roman, M. T. Fernándezdíaz and J. A. Alonso, Phys. Chem. Chem. Phys., 2010, 12, 13616.

40 L. V. Kovalev, M. V. Yarmolich, M. L. Petrova, J. Ustarroz, H. A. Terryn, N. A. Kalanda and M. L. Zheludkevich, ACS Appl. Mater. Interfaces, 2014, 6, 19201.
41 H. Jalili, N. F. Heinig and K. T. Leung, Phys. Rev. B: Condens. Matter Mater. Phys., 2009, 79, 174427.

42 D. D. Sarma, S. Ray, K. Tanaka, M. Kobayashi, A. Fujimori, P. Sanyal, H. Krishnamurthy and C. Dasgupta, Phys. Rev. Lett., 2007, 98, 157205.

43 P. Anil Kumar and D. D. Sarma, Appl. Phys. Lett., 2012, 100, 2472.

44 R. P. Panguluri, S. Xu, Y. Moritomo, I. V. Solovyev and B. Nadgorny, Appl. Phys. Lett., 2009, 94, 012501.

45 M. Wojcik, E. Jedryka, S. Nadolski, D. Rubi, C. Frontera, J. Fontcuberta, B. Jurca, N. Dragoe and P. Berthet, Phys. Rev. B, 2005, 71, 104410.

46 Q. Zhang, Y. G. Xiao, Z. F. Xu, G. Y. Liu and J. B. Li, J. Solid State Chem., 2010, 183, 2432.

47 S. W. Cheong and H. Y. Hwang, ChemInform, 2001, 32, DOI: 10.1002/chin.200103237. 